This is the author's version of an article published in Engineering Optimization. Changes were made to this version by the publisher prior to publication. DOI: http://dx.doi.org/10.1080/0305215X.2016.1208191

\title{
Design optimization of ironless multi-stage axial-flux permanent magnet generators for offshore wind turbines
}

Zhaoqiang Zhang ${ }^{\mathrm{a}}$, Robert Nilssen ${ }^{\mathrm{b}}$, S. M. Muyeen ${ }^{\mathrm{c}^{*}}$, Arne Nysveen ${ }^{\mathrm{b}}$, and Ahmed Aldurra $^{\mathrm{c}}$

${ }^{a}$ Idea Converter AS, Trondheim, Norway; ${ }^{b}$ Norwegian University of Science and Technology, NO-7491, Trondheim, Norway; ${ }^{c}$ The Petroleum Institute, P. O. Box 2533, Abu Dhabi, United Arab Emirates

Corresponding author. Email: smmuyeen@pi.ac.ae

\section{Acknowledgments}

This work was supported by Norwegian Research Centre of Offshore Wind Technology (NOWITECH). 


\title{
Design optimization of ironless multi-stage axial-flux permanent magnet generators for offshore wind turbines
}

\begin{abstract}
Direct-driven ironless-stator machines have been reported to have low requirement to the strength of the supporting structures. This feature is attractive for offshore wind turbines where lightweight generators are preferred. However, to produce sufficient torque, ironless generators are normally designed at large diameters, which can be a challenge to the machine structural reliability. Ironless Multi-Stage Axial-Flux Permanent Magnet Generator (MS-AFPMG) embraces the advantages of ironless machines but has relatively small diameter. The objective of this article is to present the design optimization and performance investigation of the ironless MS-AFPMG. An existing design strategy, which employs 2D and 3D static finite element analyses and genetic algorithm for machine optimization, is improved with the aim to reduce the calculation load and calculation time. This improved design strategy is used to investigate the optimal ironless MS-AFPMG. Some intrinsic features of this kind of machine are revealed.
\end{abstract}

Keywords: Genetic algorithm; ironless multi-stage axial-flux permanent magnet generators; machine optimization; offshore wind turbines

\section{Introduction}

The offshore wind power application demands high-power and lightweight generators for minimizing the cost. A promising candidate that can meet this need is the large-diameter ironless machine, which is characterized by the lightweight supporting structures, because of the negligible normal stress between the stator and the rotor (Spooner et al. 2005, Mueller and McDonald 2009). However, as the outer diameter goes large, the challenges in structural design arise, and the machine reliability may be reduced. In addition, the installation, operation and maintenances for large-diameter structures are of the concern. An alternative solution to the axial-flux version of this ironless concept is to reduce the outer diameter but have multiple stages in the axial direction, namely ironless Multi-Stage Axial-Flux Permanent Magnet Generator (MS-AFPMG). 
Known with different names (multi-disc, multi-stack, multi-layered etc.), MSAFPMGs (mainly iron-cored machines) have been used in small-power applications where the machine radial dimension is constrained and high torque density is expected, e.g. ship propulsion (Caricchi, Crescimbini, and Honorati 1999), automobile (Javadi and Mirsalim 2008), plan landing gear (Dumas, Enrici, and Matt 2012), and small wind turbine (Gerlando et al. 2011). Interests in MW and multi-MW ironless MS-AFPMGs for wind power application have emerged (Kobayashi et al. 2009, McDonald, Benatmane, and Mueller 2011, Zhang et al. 2013), because of low supporting structure weight, modularity, and fault tolerance.

As it has been reported in Javadi and Mirsalim (2008) and in Mueller, McDonald, and Macpherson (2005), the number of stages affects the choice of optimal iron-cored MS-AFPMG; furthermore, the output power of axial-flux machines is roughly proportional to the third power of the machine outer diameter (Capponi, Donato, and Caricchi 2012). Ironless MS-AFPMG will have more stages but smaller diameter than ironless single-stage (1S) AFPMG for the same rated power; therefore, the outer diameter and the number of stages are two important parameters to determine the optimal ironless MS-AFPMG. Unfortunately, the articles on the high-power ironless MS-AFPMGs have mainly focused on presenting and validating the concept (Kobayashi et al. 2009, McDonald, Benatmane, and Mueller 2011). So far, there have been no complete articles on the optimization of MS-AFPMG, though Zhang et al. (2013) presents some investigations with incomplete description of the modelling and calculation results.

The authors previously developed a design strategy in which the 2D and 3D Static Finite Element Analyses (SFEAs) were driven by a Genetic Algorithm (GA) for machine optimization. This design strategy was successfully used in the optimization of $1 \mathrm{~S}$ machines (Zhang et al. 2014), and the lab tests in a $50 \mathrm{~kW}$ ironless axial-flux permanent 
magnet generator (Figure 1) confirmed its high accuracy. However, it has the drawback of long calculation time.

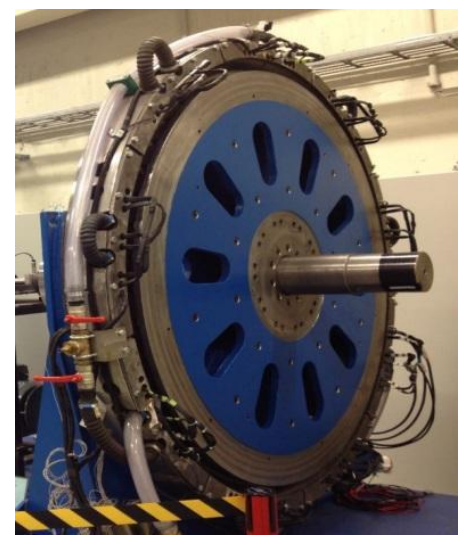

Figure 1. 50kW PM generator prototype (courtesy of SmartMotor AS).

The use of permanent magnet machines in wind energy application is getting popular nowadays due to robustness and gearless low speed operation. The general electrical scheme of variable speed permanent magnet synchronous generator used in both onshore and offshore wind farms are shown in Figure 2. In offshore industry, it is required to use big machines to extract maximum power from wind as well as reducing the installation and maintenance costs. One example for such an application is an axialflux permanent magnet based wind generator shown in Figure 1 by targeting a 10 MW class wind turbine. An attempt has been made in this work to improve the design, as low weight machine is more feasible for offshore wind turbine application. In this article, a form factor is introduced and the corresponding optimization method is upgraded to improve the calculation efficiency. GA is used for its adaptability (Wang et al. 2016) and high probability of capturing the global minima (Touat et al. 2014). This improved optimization strategy is used to investigate the effects of outer diameter and number of stages on the ironless MS-AFPMG performance. This article is organized as follows: 
Section 2 introduces the specifications and types of the studied machines. The design and optimization methods are presented in Section 3. In section 4, the optimal ironless MSAFPMGs, in terms of highest torque density, highest efficiency, and multiple objectives, are investigated with the improved optimization method; Section 5 concludes this article.

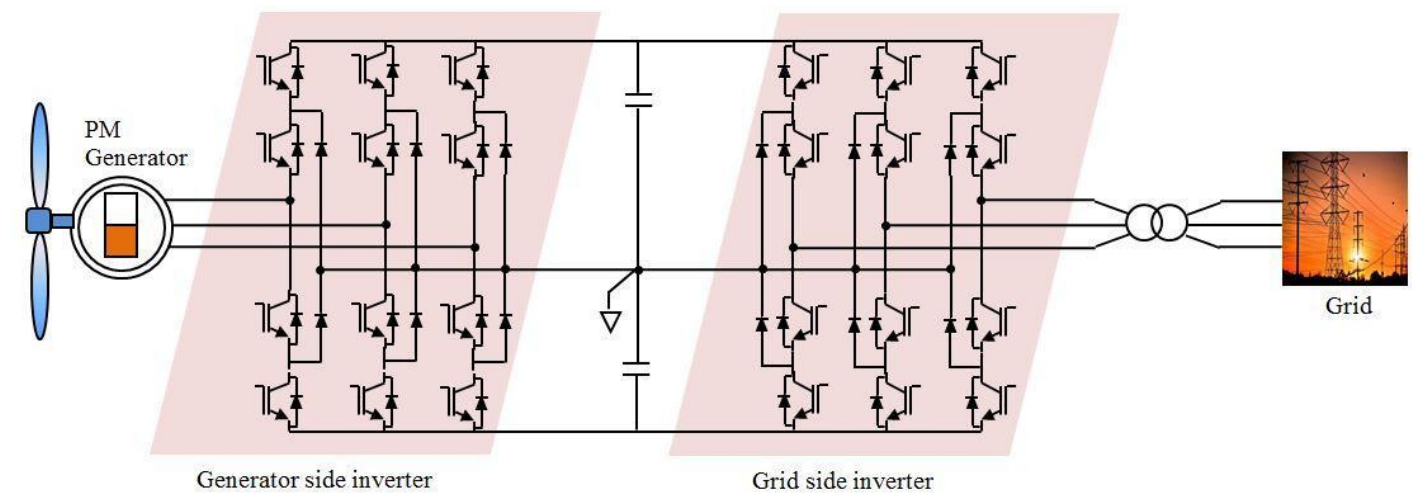

Figure 2. Electrical schematic for PM based wind generator.

\section{Machine specification and types}

The studied ironless MS-AFPMG consists of several identical stages. Each stage (Figure 3) has two rotors and one ironless stator comprising coils casted in epoxy resin. The stator is segmented to physically separated parts, and each part carries the same power.

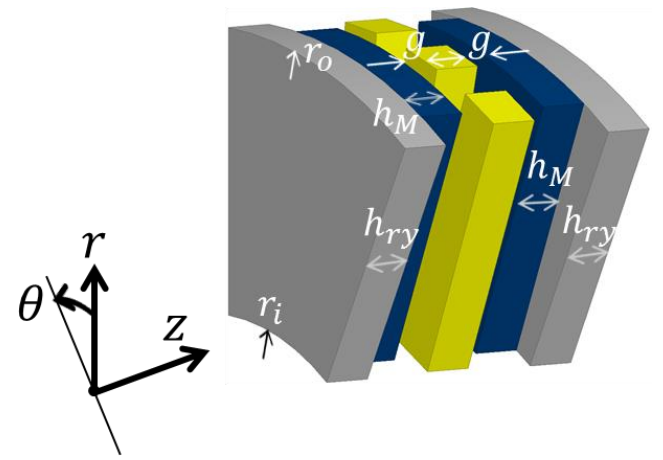

Figure 3. Machine dimension in cylindrical coordinate ( $g$ is the air gap thickness, $h_{M}$ is PM thickness, $h_{r y}$ is rotor yoke thickness, $r_{i}$ is inner radius, and $r_{o}$ is outer radius. $r, \theta$ and $z$ are the axes of the cylindrical coordinate). 
Generator specification is given in Table 1. It has 9 segments in total. The coil fill factor is low due to the use of heavily twisted Litz wires for minimizing the eddy current loss in the winding. The material specific costs are obtained from the industrial experience of the authors, and for information only. The rotor iron loss is low in this low-speed machine; solid steel is therefore used in the rotor yoke.

Table 1. Generator specification.

\begin{tabular}{|l|c|c|}
\hline \multicolumn{1}{|c|}{ Parameters } & Value & Type \\
\hline Rated power $(\mathrm{MW})$ & 10 & Constant \\
\hline Number of phases & 3 & Constant \\
\hline Rated speed $(\mathrm{rpm})$ & 12 & Constant \\
\hline Rated voltage $(\mathrm{kV})$ & 6.8 & Constant \\
\hline Total number of segments & 9 & Constant \\
\hline Coil fill factor & 0.5 & Constant \\
\hline PM specific cost $(€ / \mathrm{kg})$ & 80 & Constant \\
\hline Iron specific cost $(€ / \mathrm{kg})$ & 16 & Constant \\
\hline Copper specific cost $(€ / \mathrm{kg})$ & 27 & Constant \\
\hline Outer diameter $(\mathrm{m})$ & $10-24$ & Free variable \\
\hline Number of poles, $p$ & $90-720$ & Free variable \\
\hline Ratio of PM width to pole pitch, $k_{M}$ & $0.1-0.8$ & Free variable \\
\hline Peak of the air gap $1^{\text {st }}$ order flux density, $B_{p 1}(\mathrm{~T})$ & $0.1-0.8$ & Free variable \\
\hline Current density, $J\left(\mathrm{~A} / \mathrm{mm}^{2}\right)$ & $2-5$ & Free variable \\
\hline Air gap thickness, $g(\mathrm{~mm})$ & $0.15 \% D_{o}$ & Constraint \\
\hline PM thickness, $h_{M}(\mathrm{~mm})$ & $5-100$ & Constraint \\
\hline Rotor yoke thickness, $h_{r y}(\mathrm{~mm})$ & $5-100$ & Constraint \\
\hline Reference rotor yoke flux density, $B_{r y}^{\text {ref }}(\mathrm{T})$ & 1.7 & Constraint \\
\hline Electric load at inner diameter, $E_{L}(\mathrm{kA} / \mathrm{m})$ & 50 & Constraint \\
\hline PM type & $\mathrm{N} 42 \mathrm{SH}$ & -- \\
\hline Iron type & Solid steel & -- \\
\hline
\end{tabular}

Three types of generators are considered, namely

(1) $1 \mathrm{~S}$-AFPMG, 9 segments.

(2) Two-stage (2S) AFPMG, 9 segments per stage, 2 segments from adjacent stages are connected parallelly to the same load, and therefore, the total number of segments is equivalent to 9 . 
(3) Three-stage (3S) AFPMG, 3 segments per stage.

\section{Design and optimization method}

This section presents the improvement of an existing design strategy, with the aim of reducing the calculation load and shortening the calculation time.

\subsection{Limitation of the existing method}

Zhang et al. (2014) developed a design strategy in which the 2D and 3D SFEAs were driven by a GA for machine optimization. This design strategy requires the accurate calculation of a leakage coefficient $\left(k_{l e}\right)$, which is used to calculate the fundamental backEMF and given below

$$
k_{l e}=\frac{\int_{r_{i}}^{r_{o}} B_{p 1}(r) r d r}{B_{p 1} l r_{a}}
$$

where $l$ is coil active length, and $r_{a}=\sqrt{r_{i} r_{o}}$. At the radius $r_{a}$, a plane called fieldcomputation plane is defined, because it is at this plane 2D SFEA is conducted to size the machine.

Practically, it is impossible to perform the integration in (1) in the postprocessing of a SFEA, which does not contain the information about the harmonic components of the air gap field. A feasible way is to pick up evenly distributed lines in the air gap along the coil active length, then apply FFT to the field of each line, and finally sum up all the results. To ensure an accurate $k_{l e}$, a large amount of FFTs are required in postprocessing, which is cumbersome and time-consuming. In addition, the calculation of $k_{l e}$ requires multiple iterations (mainly 3D SFEAs) for obtaining a converged $k_{l e}$. The number of iterations affects the total calculation time, and depends on how close the initial guess of $k_{l e}$ is to its practical value. However, in a multidimensional optimization, it is not easy to 
give a good estimation of the initial value so that the number of iterations is as small as possible. Therefore, for improving the calculation efficiency, it is necessary to investigate other methods.

\subsection{Proposed method and form factor}

According to Faraday's law, the transient back-EMF for a $N$-turn full-pitch coil is given by

$$
e=N \frac{d \phi}{d t}=N \sum_{1}^{n} \Phi_{p k} \omega_{k} \sin \left(\omega_{k} t+\varphi_{k}\right)
$$

where $\phi$ is the total transient flux penetrating a one-turn coil, $t$ is time, $n$ is the total harmonics considered, $\Phi_{p k}$ is the peak value of the $k^{\text {th }}$ flux harmonic, $\omega_{k}$ is electrical angular velocity, and $\varphi_{k}$ is phase angle.

To calculate the fundamental back-EMF, the corresponding $\Phi_{p 1}$ is needed. Normally this requires multiple SFEAs or a transient FEA to obtain the $\phi-t$ curve, then a FFT is conducted to extract $\Phi_{p 1}$. However, this process is also time-consuming.

In this study, a method is proposed to calculate $\Phi_{p 1}$ with reduced calculation load and calculation time. Three calculation steps are needed.

Step 1: calculate the form factor $\left(k_{f f}\right)$ at the field-calculation plan. $k_{f f}$ is defined in (3). Its numerator calculates the flux (for a unit radial length) corresponding to the fundamental harmonic, and the denominator calculates the total flux (for a unit radial length). Therefore, the form factor, actually represents the percentage of fundamental flux in the total flux in the field-calculation plan. To get $B_{p 1}$, one FFT operation is required to the field distribution along the field-calculation line (Figure 4). 


$$
k_{f f}=\frac{\int_{\frac{\theta p}{2}}^{\frac{\theta p}{2}} B_{p 1} \cos \left(\frac{\pi}{\theta_{p}} \theta\right) r_{a} d \theta}{\int_{-\frac{\theta p}{2}}^{\frac{\theta p}{2}} B_{Z} r_{a} d \theta}=\frac{\frac{2}{\pi} B_{p 1} \theta_{p}}{\int_{-\frac{\theta p}{2}}^{\frac{\theta p}{2}} B_{Z} d \theta}
$$

where $\theta_{p}$ is the pole pitch in radians, $B_{z}$ is the $z$-component of the flux density for a space point.

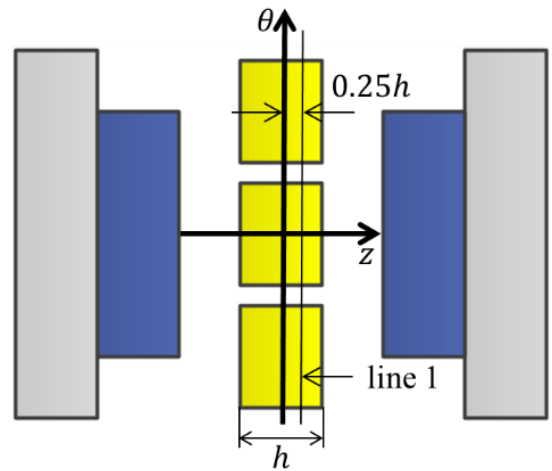

Figure 4. Field-calculation line (line 1) in the field-calculation plane ( $h$ is the thickness of the coil).

Step 2: do a 2D field integration under one pole over the conductor plane cut by the field-calculation line, which gives the maximum flux $\left(\Phi_{p}\right)$ penetrating the coil. The mathematical expression of this integration is

$$
\left.\Phi_{p}\right|_{(z=0.25 h)}=\int_{r_{i}}^{r_{o}} \int_{-\frac{\theta p}{2}}^{\frac{\theta p}{2}} B_{z} r d \theta d r
$$

Step 3: assume all the planes (parallel to the field-calculation plane) along the coil active length have the same form factor in the air gap field, then the fundamental flux can be given by

$$
\Phi_{p 1}=\left.k_{f f} \Phi_{p}\right|_{(z=0.25 h)} .
$$




\subsection{Upgraded design and optimization strategy}

The upgraded design and optimization procedure is illustrated in Figure 5. The machine sizing and parameter calculation are directly conducted in 2D and 3D SFEAs. Two iteration loops are used, one for voltage drop ratio $\kappa$ (the magnitude difference of rated back-EMF and rated voltage), and another for $k_{f f}$. This method is driven by the GA of the Matlab optimization toolbox.

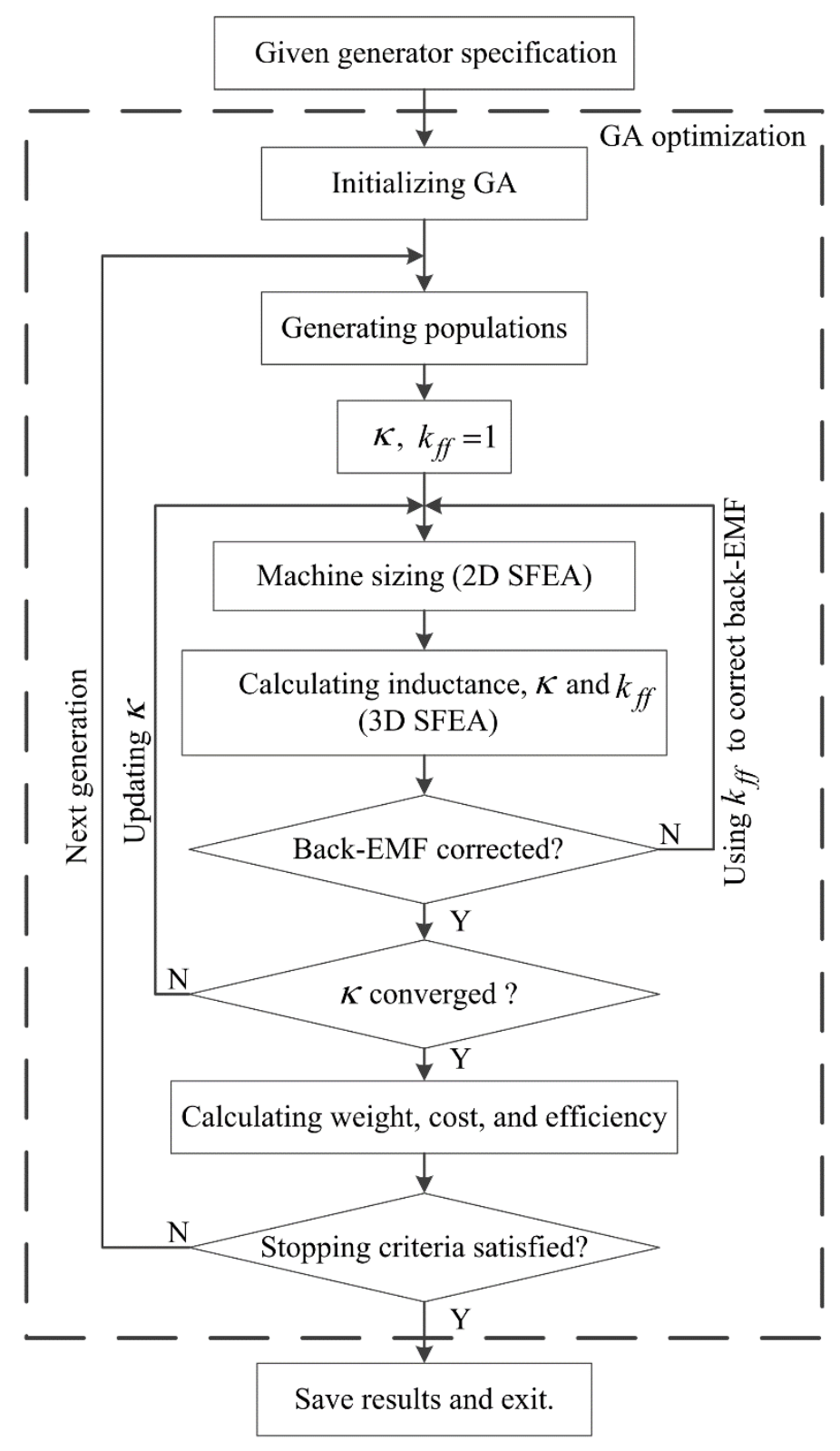

Figure 5. Design procedure ( $\kappa$ is voltage drop ratio, $k_{f f}$ is form factor). 
For a single-objective optimization, the problem is formulated as

$$
f_{o p t}=\min _{o b j}\left(D_{o}, p, k_{M}, B_{p 1}, J\right)
$$

where $f_{o b j}$ is the optimization objective, which can be the negative value of torque density or negative efficiency. Torque density and efficiency are defined in (7) and (8).

$$
\begin{aligned}
& T_{D}=\frac{T}{M_{b i}+M_{p m}+M_{c u}} \\
& \eta=\frac{P_{o}}{P_{o}+P_{b i}+P_{p m}+P_{c u}+P_{w f}},
\end{aligned}
$$

where $M_{b i}, M_{p m}, M_{c u}, P_{o}, P_{b i}, P_{p m}, P_{c u}$, and $P_{w f}$ are back iron weight, permanent magnet weight, copper winding weight, machine output power, back iron loss, permanent magnet loss, copper winding loss, windage and friction loss, respectively. This fitness function is subjected to the following constraints

$$
\begin{gathered}
g\left(D_{o}, p, k_{M}, B_{p 1}, J\right)=0.001 D_{o} \\
5 \leq h_{M}\left(D_{o}, p, k_{M}, B_{p 1} J\right) \leq 100 \\
5 \leq h_{r y}\left(D_{o}, p, k_{M}, B_{p 1}, J\right) \leq 100 \\
B_{r y}^{r e f}\left(D_{o}, p, k_{M}, B_{p 1}, J\right)=1.7 \\
E_{L}\left(D_{o}, p, k_{M}, B_{p 1}, J\right)=50 .
\end{gathered}
$$

For a multi-objective optimization where torque density, efficiency, and cost are all taken into account (see section 4), the problem is managed and solved in this way. Torque density and efficiency are calculated sequentially, and compared to reference 
setting points. If they are greater than the reference setting points, then the cost of these populations are set to a default large number, so that they cannot be favoured and will have low impact on producing the next generations (This managing theme relies on the fact that optimizations with genetic algorithm are not gradient-dependent). If they are less than the reference setting points, then the machine cost is calculated. The machine with the lowest cost will be the optimal one in that generation.

This is a mixed-integer (pole number must be an integer) optimization problem. Solving mixed-integer optimization is subject to limitations when choosing the proper mutation and crossover functions. Several calculation tests show that, in Matlab optimization toolbox, the default setting of mutation and crossover functions gives the best performance in term of computational efficiency. Therefore, these mutation and crossover functions are used throughout the whole calculations.

\subsection{Performance of the improved method}

To investigate the performance of the improved method, a random design case is used. Its task is to dimension a generator that meets the specifications in Table 1, with the given input in the upper part of the Table 2. Note that the aspect ratio is the ratio of machine axial length to its outer diameter, $\sigma$ is the ratio of inner radius to outer radius, torque density is the rated torque divided by the weight of active parts (winding, PM, and rotor iron), and power cost is the cost of the active parts divided by the rated power.

The calculation results (lower part of the Table 2) show that both methods can dimension the generator properly, and their design results are close to each other. However, with the improved method, the numbers of 2D and 3D SFEAs conducted are reduced, as well as the total calculation time. 
Table 2. Method comparison.

\begin{tabular}{|c|c|c|c|}
\hline & Parameters & Improved method & $\begin{array}{c}\text { Method } \\
\text { in Zhang et al. (2014) }\end{array}$ \\
\hline \multirow{7}{*}{$\begin{array}{l}\text { Calculation } \\
\text { input }\end{array}$} & Number of stages & \multicolumn{2}{|r|}{ 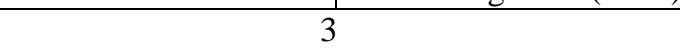 } \\
\hline & $D_{o}(\mathrm{~m})$ & \multicolumn{2}{|c|}{10} \\
\hline & $k_{M}$ & \multicolumn{2}{|c|}{0.73} \\
\hline & $B_{p 1}(\mathrm{~T})$ & \multicolumn{2}{|c|}{0.73} \\
\hline & $p$ & \multicolumn{2}{|c|}{138} \\
\hline & $J\left(\mathrm{~A} / \mathrm{mm}^{2}\right)$ & \multicolumn{2}{|c|}{2} \\
\hline & Initial $k_{f f}$ and $k_{l e}$ & $k_{f f}=1$ & $k_{l e}=1$ \\
\hline \multirow{9}{*}{$\begin{array}{l}\text { Calculation } \\
\text { result }\end{array}$} & Aspect ratio & 0.127 & 0.124 \\
\hline & $\sigma$ & 0.794 & 0.777 \\
\hline & Torque density $(\mathrm{Nm} / \mathrm{kg})$ & 37.06 & 35.60 \\
\hline & Efficiency (\%) & 96.9 & 96.9 \\
\hline & Power cost $(€ / \mathrm{W})$ & 0.89 & 0.93 \\
\hline & Calculated $k_{f f}$ and $k_{l e}$ & $k_{f f}=0.983$ & $k_{l e}=0.954$ \\
\hline & Number of 2D SFEAs & 271 & 378 \\
\hline & Number of 3D SFEAs & 6 & 8 \\
\hline & Total calculation time & $16.19 \mathrm{~min}$. & 20.83 min. \\
\hline
\end{tabular}

\subsection{Population size}

Table 1 and 2 show that one integer (pole number) is used as the free variable. For this mixed integer optimization problem with five free variables, Matlab suggests the population size to be 50. This suggestion works fine, and normally after several generations, the fitness function will try to converge. However, at this moment, one may notice that, even though there is not much change in the value of the fitness function, the position (values of free variables) where the fitness function is obtained does vary a lot.

In this study, the interest is not just to investigate the optimal designs, but also the positions corresponding to the optimal designs. Therefore, having enough populations is important for this study. In practical optimization, it is empirically decided to set the number of populations to 100 (stop criterion), so that the position (values of free variables) when the optimal design is obtained does not vary much.

\section{Optimization results}

The improved method is used to find the optimal designs in terms of highest torque 
density, highest efficiency, and multiple objectives. Note that the first point on the left side of each curve (Figure 6 and Figure 7) shows the first feasible design as the outer diameter grows with a step of $2 \mathrm{~m}$, and for each machine type, the optimal designs at five different diameters are investigated.

Four workstations (each has $2 *$ Xeon X5687 3.6GHz, 24GB RAM) were used, and it took around two weeks to complete all the calculations.

\subsection{Optimal designs in term of highest torque density}

As depicted in Figure 6(a), the torque density increases as the diameter grows, this meets the expectation that large-diameter machines normally produce more torque. At small diameters (lower than $16 \mathrm{~m}$ ), the more number of stages, the higher torque density the machines have.

The other parameters are given in Figure 6(b)-(j). Machines with fewer number of stages present higher efficiency. As the diameter grows, the machine power cost decreases, and machines with fewer number of stages have higher power cost at low diameters. Above observations suggest that, in term of optimizing the torque density, it is better to build $1 \mathrm{~S}$ machine at large diameter; if the outer diameter is constrained to a small value, MS solutions outperform the $1 \mathrm{~S}$ solution while having lower efficiency and lower post cost.

$k_{M}$ and $B_{p 1}$ decrease as the diameter grows, however, machines with fewer number of stages tend to have higher $k_{M}$ and $B_{p 1}$. Note that $k_{M}$ is very low. It is because this is a single-objective optimization and the GA tries to search the designs that use more copper than iron and have high current loading. Prioritizing the reduction of the stator mass seems an effective way to improve torque density in this optimization case. Pole 
number increases as the diameter grows. Current density for $1 \mathrm{~S}$ machine increases to its upper boundary $\left(5 \mathrm{~A} / \mathrm{mm}^{2}\right)$, whereas it stays close to the upper boundary for MS machines. This can partly explain why $1 \mathrm{~S}$ machine has higher efficiency than MS machines. As the diameter grows, the aspect ratio decreases and $\sigma$ increases, indicating that machines become more like rings. Also note that as an important indication of the torque density, $\sigma$ varies with the application (e.g. 0.7-0.8 recommended for ship propulsion, Caricchi, Crescimbini, and Honorati 1999). Figure 6(j) reveals the diameterdependency of $\sigma$, which can be greater than 0.9 in this design case. The increase in the voltage drop ratio implies the increase in the reactance, and thus the decrease in the power factor.

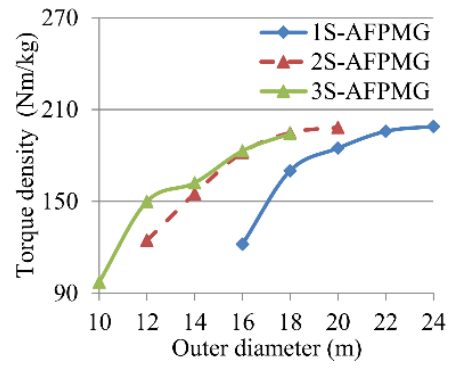

(a)

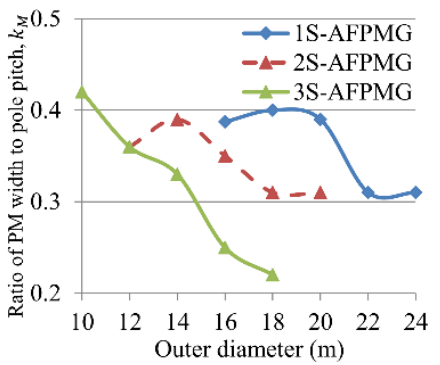

(d)

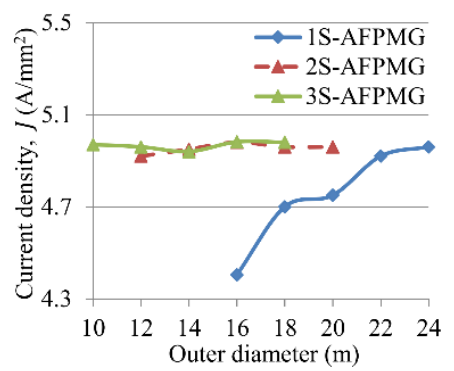

(g)

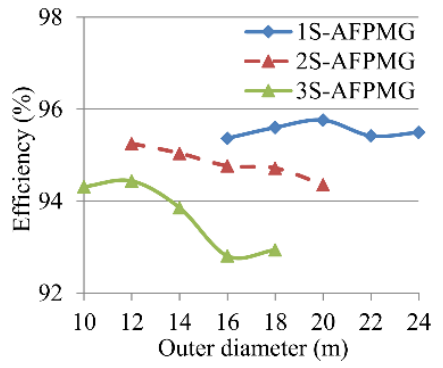

(b)

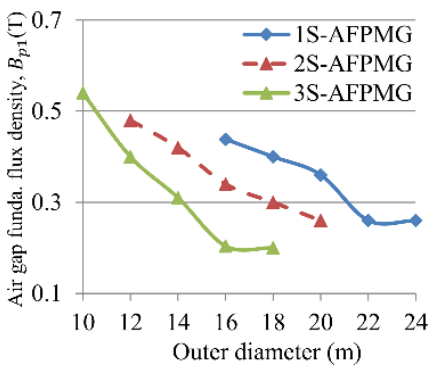

(e)

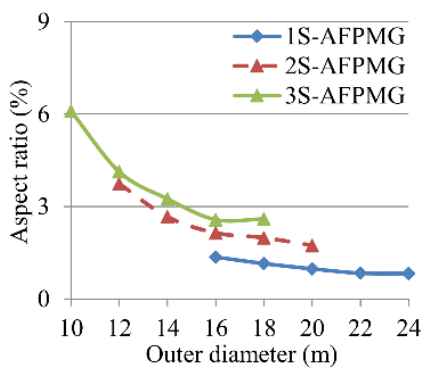

(h)

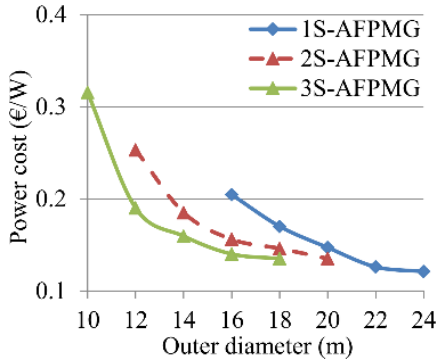

(c)

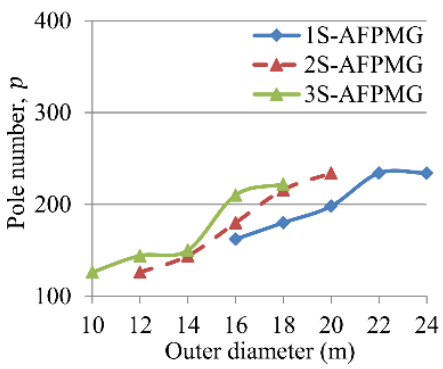

(f)

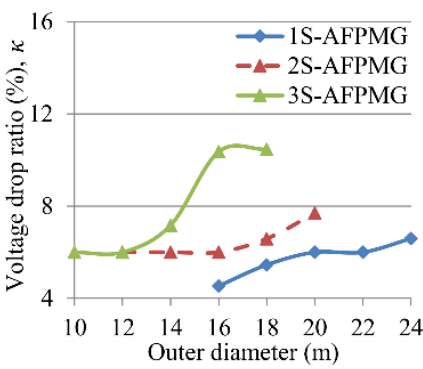

(i) 


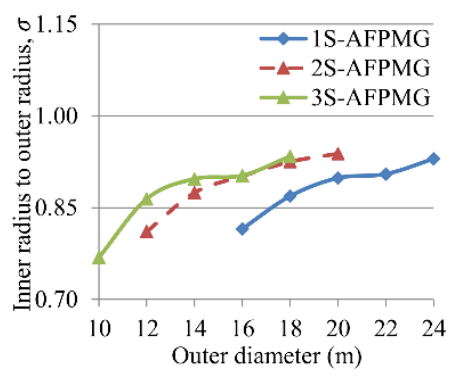

(j)

Figure 6. Optimal designs in term of highest torque density.

\subsection{Optimal designs in term of highest efficiency}

As shown in Figure 7(a), the efficiency does not vary much with the increase in the diameter, whereas the more number of stages, the lower efficiency the machine has. This is mainly due to the higher copper loss in the MS machines.

The other parameters are given in Figure 7(b)-(j). As the diameter increases, the torque density increases and the power cost decreases. Above observations suggest that, in term of optimizing the efficiency, it is better to build a $1 \mathrm{~S}$ machine at a large diameter. Even if the diameter is constrained to a small value, machines with fewer stages still outperform those with more number of stages.

As the diameter grows, $B_{p 1}$ decreases, whereas $k_{M}$ almost stays constant around 0.77, which is close to the result (0.78) in Wang et al. (2005) where the machine efficiency was optimized with Powell's method. Pole number increases in all three machines. Current density for all three machines remains close to its lowest boundary ( $2 \mathrm{~A} / \mathrm{mm} 2)$, which helps to reduce copper loss. The aspect ratio and $\sigma$ present the same trend as the previous case. Voltage drop ratio for $1 \mathrm{~S}$ machine keeps almost stable, whereas it increases for MS machines. 


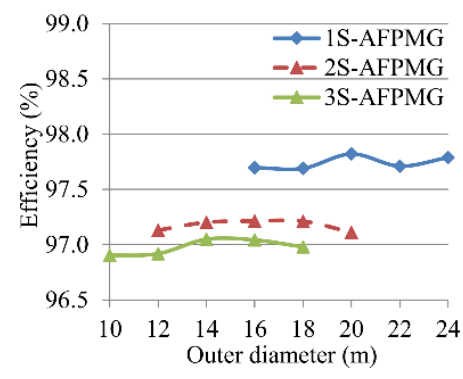

(a)

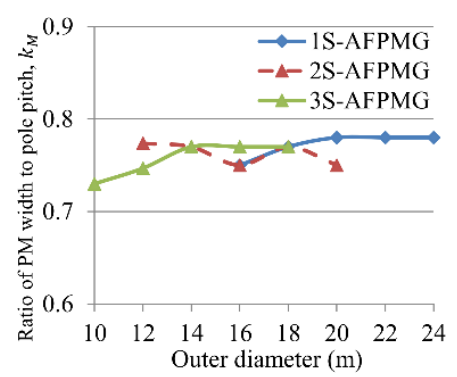

(d)

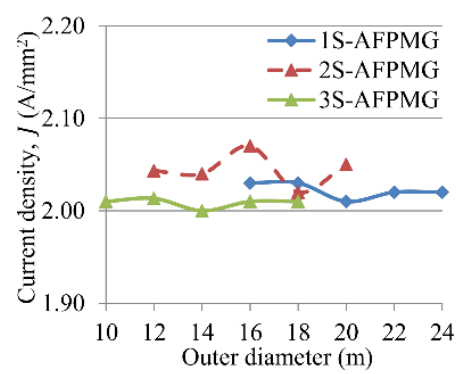

(g)

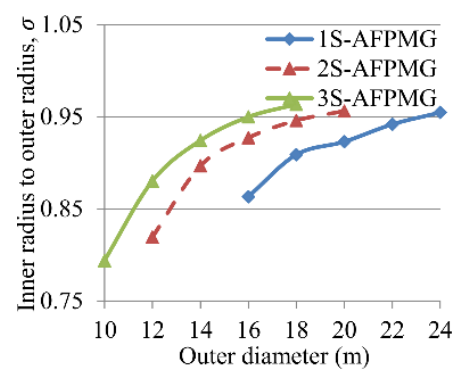

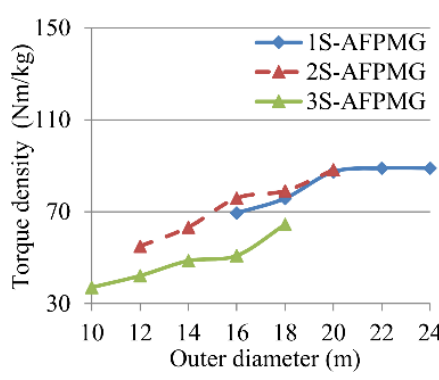

(b)

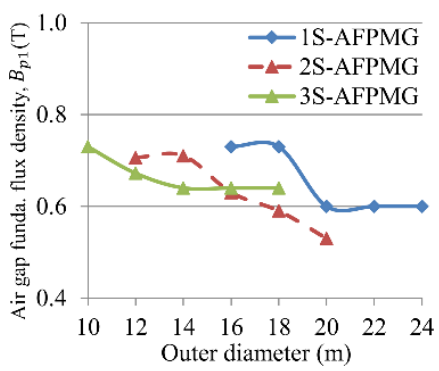

(e)

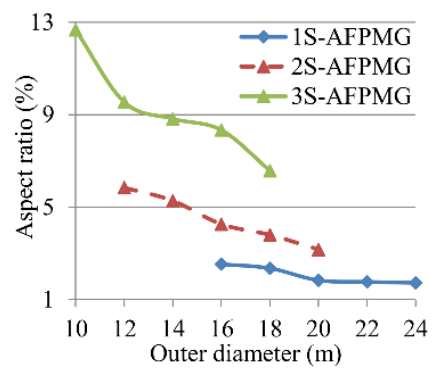

(h)

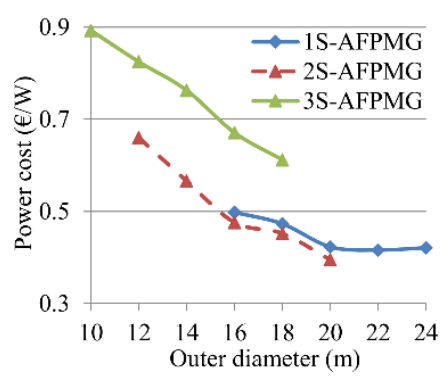

(c)

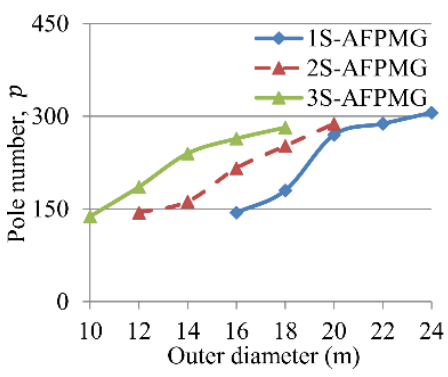

(f)

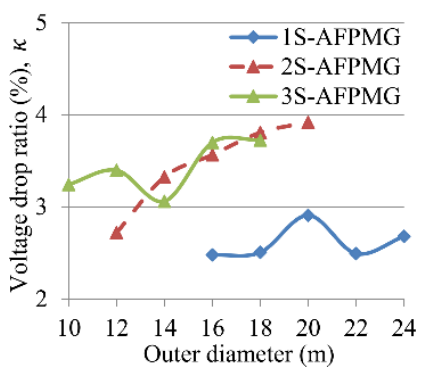

(i)

(j)

Figure 7. Optimal designs in term of highest efficiency. 


\subsection{Optimal designs in term of multiple objectives at outer diameter $18 \mathrm{~m}$}

Practically, the determination of the optimal machines involves the trade-off of multiple objectives. The formulation of a multi-objective fitness function normally depends on the specific application and the optimization algorithm (Gao et al. 2012 and Wang et al. 2013). In this study, the optimal machines that satisfy the following objectives at outer diameter $18 \mathrm{~m}$ are investigated:

(1) The active weight is lower than 60 tons.

(2) The efficiency is greater than $95 \%$.

(3) The power cost is the lowest.

The best designs from all three machines are given in Table 3, which shows that the optimal design is a $2 \mathrm{~S}$ machine because of the lowest power cost. This optimal design also has the highest torque density, but its efficiency is lower than that for the best $1 \mathrm{~S}$ machine. This optimization case implies that there is no general rule to follow, i.e., prioritizing $1 \mathrm{~S}$ solution or MS solutions, if multiple objectives come into the consideration.

Table 3. Optimal designs in term of multiple objectives.

\begin{tabular}{|c|c|c|c|}
\hline & 1S-AFPMG & 2S-AFPMG & 3S-AFPMG \\
\hline$k_{M}$ & 0.4 & 0.42 & 0.39 \\
\hline$B_{p 1}(\mathrm{~T})$ & 0.4 & 0.37 & 0.29 \\
\hline$p$ & 180 & 180 & 204 \\
\hline$J\left(\mathrm{~A} / \mathrm{mm}^{2}\right)$ & 4.70 & 4.65 & 3.38 \\
\hline Aspect ratio $(\%)$ & 1.15 & 2.08 & 2.82 \\
\hline$\kappa(\%)$ & 5.45 & 7.28 & 8.85 \\
\hline$\sigma$ & 0.87 & 0.93 & 0.94 \\
\hline Torque density $(\mathrm{Nm} / \mathrm{kg})$ & 170.08 & 171.69 & 148.94 \\
\hline Efficiency $(\%)$ & 95.6 & 95.1 & 95.1 \\
\hline Power cost $(€ / \mathrm{W})$ & 0.17 & 0.16 & 0.18 \\
\hline
\end{tabular}




\subsection{General remarks}

Following general conclusions can be drawn from the above three investigations:

(1) The diameter and number of stages significantly affect the performance of ironless MS-AFPMG

(2) If there is no dimensional constraint, it is always better to design a machine at a larger diameter for higher torque density and lower power cost. But of course, the weight of the inactive parts (supporting structures) is not taken into account.

(3) If there are dimensional constraints, the number of stages will affect the determination of the optimal machines at a fixed diameter. Generally, machines with more number of stages tend to have lower efficiency because of more copper losses.

(4) In some cases, e.g., there is only one design objective for a small diameter, or there are multiple objectives, MS machines may outperform 1S machine.

(5) Machines with high torque density tend to have high current density, small $k_{M}$, and low air gap field, whereas machines with high efficiency tend to have low current density, large $k_{M}$, and high air gap field.

(6) Note that similar optimization results for 1S-AFPMG are reported in Zhang et al. (2014); however, compared to the results in this study, the positions (values of free variables) where the optimization results are obtained are different. This implies the importance of having enough populations and generations in this kind of mixed integer GA-based optimizations. 


\section{Conclusions}

This article introduced a form factor to reduce the calculation load and shorten the calculation time of an existing design and optimization strategy. This upgraded strategy, which employs 2D and 3D SFEAs and GA for machine optimization, was used to investigate the optimal ironless MS-AFPMGs in terms of highest torque density, highest efficiency, and multiple objectives.

It has been shown that, direct-driven ironless MS-AFPMG normally has low $B_{p 1}$, low aspect ratio, large pole number, and high $\sigma$, whereas $k_{M}$ and current density varies with the fitness function. Without considering the inactive weight, it is always preferred to build a large-diameter ironless MS-AFPMG. However, if there are dimensional constraints, the MS solutions may outperform 1S solution. When the design has to deal with multiple objectives, the optimization becomes complicated, and the case-by-case investigation is needed.

Structural and thermal analyses were out of the scope of this study. Nonetheless, it should be pointed out that, maintaining the structural reliability and providing proper cooling arrangement are two crucial aspects in offshore wind turbines, which should be taken into account in the system design, and as a result, the above investigation results may be affected.

\section{Acknowledgments}

This work was supported by Norwegian Research Centre of Offshore Wind Technology (NOWITECH). 


\section{References}

Caricchi, F., Crescimbini, F., and Honorati, O. 1999. "Modular Axial-flux Permanent-magnet Motor for Ship Propulsion Drives.” IEEE Transactions on Energy Conversion 14 (3): 673-679.

Capponi, F. G., Donato, G. D., and Caricchi, F. 2012. "Recent Advances in Axial-flux Permanent-magnet Machine Technology.” IEEE Transactions Industrial Applications 48 (6): 2190-2205.

Dumas, F., Enrici, P., and Matt, D. 2012. "Design and Comparison of Two Multi-disc Permanent Magnet Motors for Aeronautical Application.” International Conference of Electrical Machines. IEEE Publications, pp. 647-652, Marseille, France.

Gao, X. Z., Wang, X., Ovaska, S. J., and Zenger, K. 2012. “A Hybrid Optimization Method of Harmony Search and Opposition-based Learning.” Engineering Optimization 44 (8): 895-914.

Gerlando, A. D., Foglia, G., Iacchetti, M. F., and Perini, R. 2011. “Axial Flux PM Machines with Concentrated Armature Windings: Design Analysis and Test Validation of Wind Energy Generators.” IEEE Transactions on Industrial Electronics 58 (9): 3795-3805.

Javadi, S., and Mirsalim, M. 2008. “A Coreless Axial-flux Permanent-magnet Generator for Automotive Applications.” IEEE Transactions on Magnetics 47 (12): 4591-4598.

Kobayashi, H., Doi, Y., Miyata, K., and Minowa, T. 2009. "Design of the Axial-flux Permanent Magnet Coreless Generator for the Multi-megawatts Wind Turbine.” European Wind Energy Conference, Marseille, France, March 16-19.

McDonald, A. S., Benatmane, M., and Mueller, M. A. 2011. “A Multi-stage Axial Flux Permanent Magnet Machine for Direct Drive Wind Turbines.” IET Renewable Power Generation Conference, Edinburgh, UK, September 6-8.

Mueller, M. A., McDonald, A. S., and Macpherson, D. E. 2005. "Structural Analysis of Low Speed Axial-flux Permanent-magnet Machines.” IEE Proceeding - Electric Power Applications 152 (6): 1417-1426.

Mueller, M. A., and McDonald, A. S. 2009. "A Lightweight Low-speed Permanent Magnet Electrical Generator for Direct-drive Wind Turbines.” Wind Energy 12 (8): 768-780.

Spooner, E., Gordon, P., Bumby, J. R., and French, C. D. 2005. "Lightweight Ironless-stator PM Generators for Directdrive Wind Turbines." IEE Proceeding - Electric Power Applications 152 (1): 17-26.

Touat, N., Benseddiq, N., Ghoul, A., and Rechak, S. 2014. “An accelerated pseudogenetic algorithm for dynamic finite element model updating." Engineering Optimization 46 (3): 340-360.

Wang, G., Chen, J., Cai, T., and Xin, B. 2013. "Decomposition-based Multi-objective Differential Evolution Particle Swarm Optimization for the Design of a Tubular Permanent Magnet Linear Synchronous Motor.” Engineering Optimization 45 (9): 1107-1127.

Wang, R., Kamper, M. J., Westhuizen, K. V. D., and Gieras, J. F. 2005. “Optimal Design of a Coreless Stator Axial Flux Permanent-magnet Generator.” IEEE Transactions on Magnetics 41 (1): 55-64. 
Wang, X., Shi, Y., Ding, D., Gu, X. 2016. “Double Global Optimum Genetic Algorithm-particle Swarm Optimizationbased Welding Robot Path Planning.” Engineering Optimization 48 (2): 299-316.

Zhang, Z., Matveev, A., Nilssen, R., and Nysveen, A. 2013. "Ironless Multi-stage Axial-flux Permanent Magnet Generator for Offshore Wind Power Application" $12^{\text {th }}$ Joint MMM/Intermag Conference, Chicago, USA, January $14-18$.

Zhang, Z., Matveev, A., Nilssen, R., and Nysveen, A. 2014. “Ironless Permanent Magnet Generator for Offshore Wind Turbines.” IEEE Transactions on Industrial Applications 50 (3): 1835-1846. 